\title{
AIRBORNE TESTS OF AN OAWL DOPPLER LIDAR: RESULTS AND POTENTIAL FOR SPACE DEPLOYMENT
}

\author{
Michael Hardesty ${ }^{1}$, Sara Tucker $^{2}$, Sunil Baidar ${ }^{1}$, Mark Beubien ${ }^{3}$ \\ ${ }^{I}$ Cooperative Institute for Research in Environmental Sciences, USA, mike.hardesty@noaa.gov \\ ${ }^{2}$ Ball Aerospace, USA \\ ${ }^{3}$ Yankee Environmental Systems, USA
}

\begin{abstract}
The 532 nm Green Optical Covariance Wind Lidar (GrOAWL) was flown on a NASA WB-57 research aircraft during the summer of 2016 to validate the instrument design and evaluate wind measurement capability and sensitivity.

Comparisons with dropsondes and atmospheric models showed good agreement, demonstrating that a GrOAWL type instrument could provide high-value wind measurements from both airborne and space-based platforms
\end{abstract}

\section{INTRODUCTION}

Global wind profiles from space-based platforms are generally recognized as the most significant currently-missing observation needed to improve numerical weather predication [1]. Observing System Simulation Experiments (OSSEs) have shown that the assimilating wind data from a spacebased Doppler lidar is likely to provide a significant increase in forecast skill [2], especially in the tropics and Southern Hemisphere where observations are currently limited.

The European Space Agency Aeolus Atmospheric Dynamics Mission, scheduled for launch in late 2017 or early 2018 , will be the first lidar in space aimed at providing wind measurements. Operating in the ultraviolet, Aeolus will feature separate receiver channels to process lidar returns from both aerosol and molecular targets to measure radial wind profiles at a single fixed line of sight orthogonal to the polar orbital track.

As a follow-on to Aeolus, we have proposed the design-to-cost ATHENA-OAWL NASA Earth Venture Instrument (EVI) mission to measure two components of the wind from the International

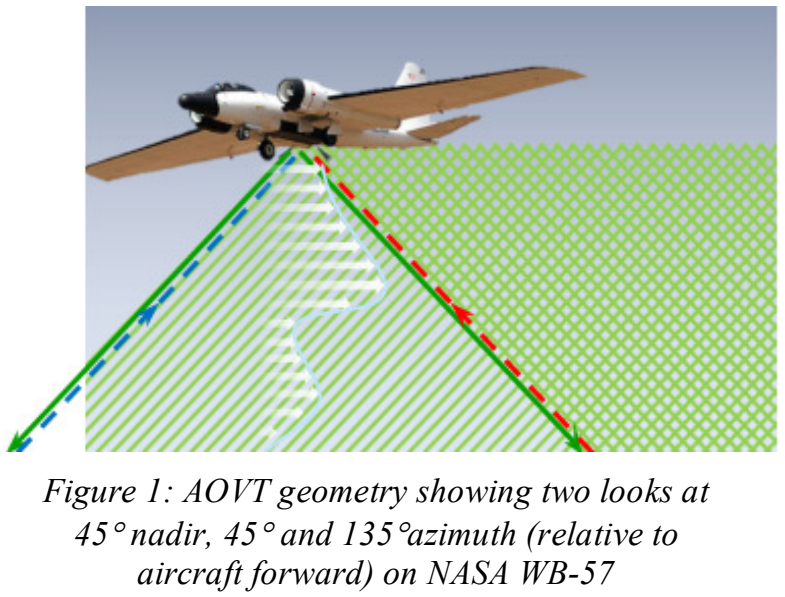

Space Station (ISS) using only aerosol/cloud atmospheric returns. Based on the Optical Autocovariance Wind Lidar (OAWL) concept, ATHENA would incorporate the 532-nm laser technology implemented on CALIPSO to reduce mission risk and cost.

During summer 2016 we carried out the ATHENAOAWL Venture-Technology (AOVT) aircraft flight program to demonstrate several key elements of the proposed ISS instrument, including instrument efficiency and wind-measuring precision, scaling to space, and two-look measurement capability. For AOVT the green OAWL (GrOAWL) instrument was mounted on a NASA WB-57, as shown in Figure 1, for a series of test flights out of Ellington Field in Houston, TX. Here we briefly describe the campaign and present some results and conclusions from the tests.

\section{GrOAWL DESCRIPTION}

The GrOAWL instrument flown during the AOVT campaign is a direct detection aerosol wind lidar built by Ball Aerospace that incorporates two 


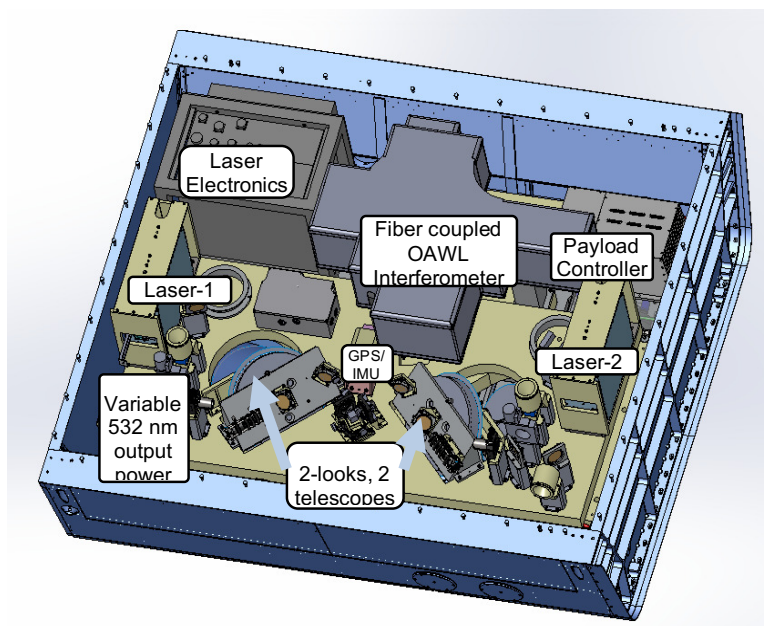

Figure 2: GrOAWL instrument shown in pressurized pallet, which mounts in the belly of the NASA WB-57 aircraft.

Nd:YAG lasers, both capable of operation at 532 and $355 \mathrm{~nm}$, and a field-widened, quadraturechannel, Mach-Zender interferometer to measure the Doppler shifts [3]. For AOVT the system operated primarily at $532 \mathrm{~nm}$ with pulse energies of $\sim 1.5 \mathrm{~mJ}$ transmitted at $200 \mathrm{~Hz}$ for each look. Two 29-cm diameter telescopes gathered the backscattered light at each look and directed it via optical fibers to the interferometer for processing. The fringes formed by the interferometer were sampled at $140 \mathrm{MHz}(1.07 \mathrm{~m})$; results were averaged both spatially and over multiple pulses to investigate wind estimate precision improvement through accumulation during the campaign. GrOAWL operated fully autonomously during each flight.

The GrOAWL system transmitted pulses alternately through each telescope, which were oriented at $45^{\circ}$ nadir angle and $45^{\circ}$ and $135^{\circ}$ azimuth angle from aircraft forward as shown in Figure 1. The entire instrument, shown in Figure 2 , was contained in a pressurized pallet that mounted in the WB-57 payload bay. Aircraftspecific designs were implemented to enable operation in a high altitude, high vibration environment; these included a vibration isolation layer underneath the instrument and kinematic mounting of the optical bench on that layer. The system was cooled by fluid loops running through external air-fluid heat exchangers. A GPS/IMU mounted on the optical bench provided information on pointing and platform motion.

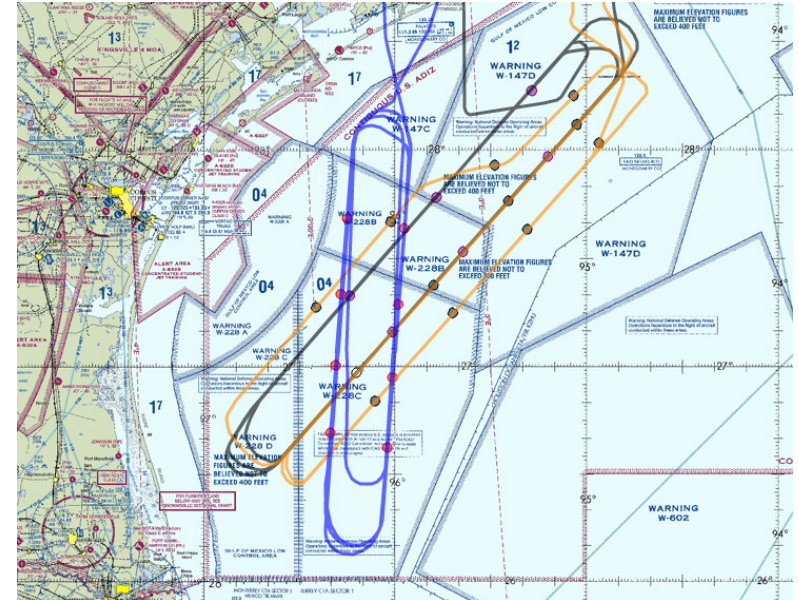

Figure 3: Flight patterns for the 3 science flights during the AOVT campaign. Dots indicate dropsonde launch positions.

\section{MEASUREMENT CAMPAIGN}

The AOVT measurement campaign consisted of 8 flights from Ellington Field, totaling approximately 38 flight hours during May-June, 2016. The flight tracks for the three science flights are shown in Figure 3. Flights were primarily over restricted areas, which provided flexibility to vary laser pulse energy, change altitude, avoid heavy clouds patterns, and minimize airspace coordination. The repeating racetrack flight patterns enabled multiple revisits of the same location, testing of the impact of multi-pulse averaging, and views of the same region of the atmosphere from opposite sides. An INMARSAT satellite link provided ground control and telemetry communication.

A key component of the campaign was the installation and utilization of the Yankee Environmental Systems dropsonde deployment system on the WB-57. A total of 21 dropsondes over three science flights were used for comparisons with the GrOAWL measurements. Radiosondes launched from Corpus Christi also provided wind data for comparison during the science flights.

\section{SCIENCE RESULTS}

After dealing with several platform-related issues such as fogging of the optical window and thermal control, we carried out three science flights during the latter part of the experiment. Figure 4 shows an example of the radial winds measured at both looks during the science flight on June 21 (blue track in 


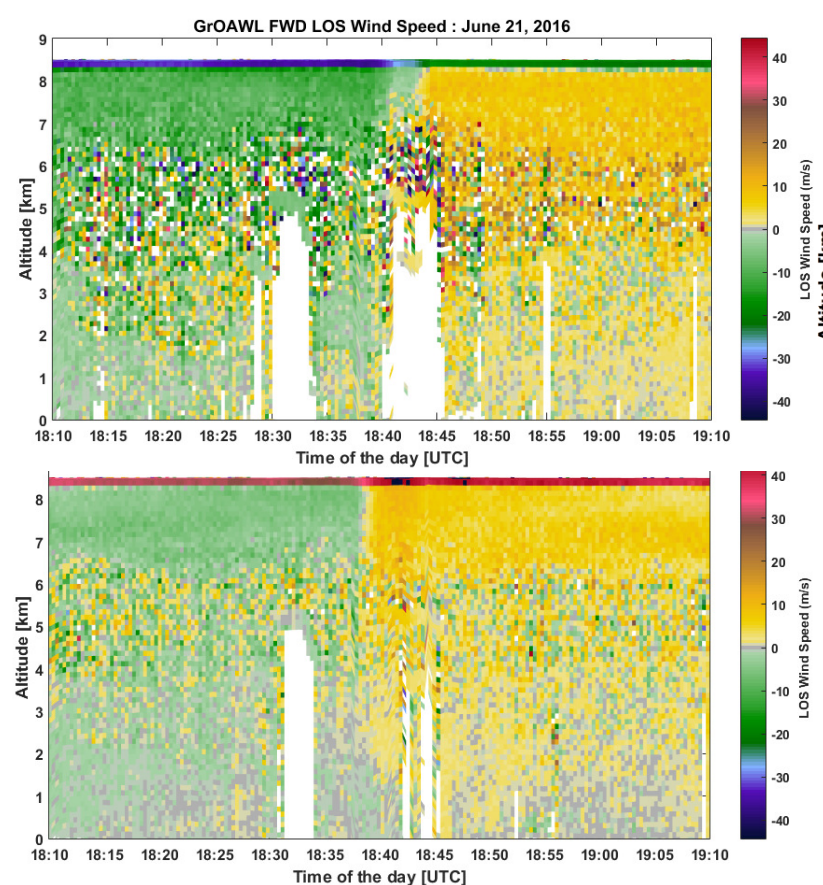

Figure 4: Radial velocity measurements for two looks on June 21. Color change at 18:38 indicates a turn. Missing data areas are due to clouds.

Figure 3) over one hour of the racetrack pattern. In Figure 4 returns were averaged over 20 seconds in time and $\sim 120 \mathrm{~m}$ vertically. Because data are collected and stored for every pulse, accumulation time can be varied to investigate wind estimate improvement. In Figure 4 the change in color is due to a turn at the end of the pattern, where the sign of the radial velocity changes. It is seen that the wind speeds for this day were quite low below $4 \mathrm{~km}$ altitude.

The profiles measured at each look were compared with the dropsonde observations. Figure 5 shows a comparison between the two looks and the dropsonde observations projected onto the line of sight for each look. Because the dropsondes fall more or less straight down from the aircraft, the lidar/sonde measurements don't overlap in space (see Figure 1). Consequently, to remove smallscale spatial variability in the wind field from the comparison we averaged the lidar wind results over 5 minutes for the comparison.

A scatterplot of the comparison between the dropsonde and the aft-pointing beam is shown in Figure 6 for the June 21 flight. Plots and comparison statistics have been generated for both looks for each flight as well as over the ensemble

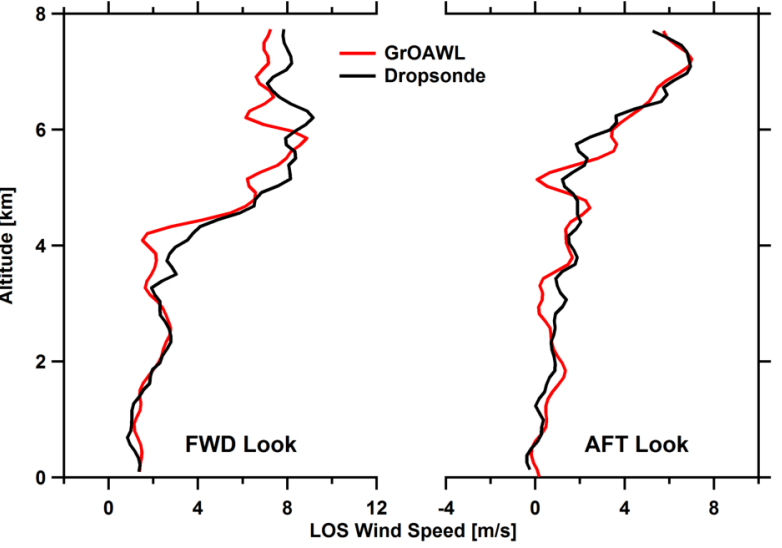

Figure 5: Example of a dropsonde comparison with two looks from GrOAWL on June 21.

of three science flights. The plot shows good agreement between the dropsonde and the GrOAWL measurements with very little bias. Bias is an important parameter for space-based observations; it must be low if the measurements are to have impact for numerical weather prediction.

Table 1 summarizes the dropsonde/GrOAWL comparisons from all three science flights (21 dropsondes in total) for three different altitude bins. Bias and standard deviation are low for all height regions and indicates excellent agreement between

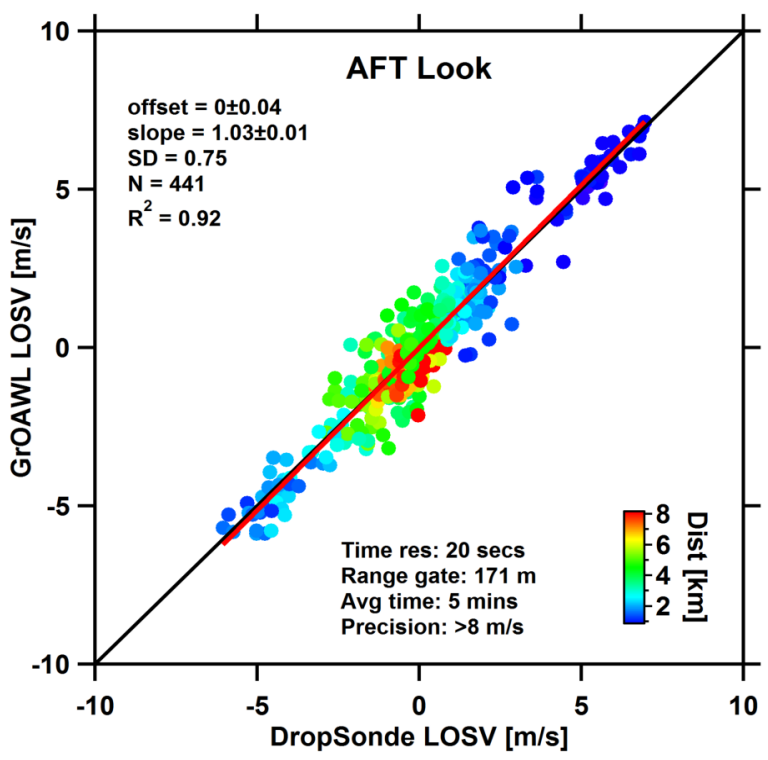

Figure 6: Scatter plot comparing GrOAWL aft look radial wind measurements with dropsonde observations. Color indicates dropsonde distance from the aircraft 
Table 1: Summary of dropsonde/GrOAWL comparisons over the science flights for three altitude regions.

\begin{tabular}{ccc}
\hline \multirow{2}{*}{ Altitude } & \multicolumn{2}{c}{$\mathrm{V}_{\text {GrOAWL }}-\mathrm{V}_{\text {dropsonde }}[\mathrm{m} / \mathrm{s}]$} \\
\cline { 2 - 3 } & FWD Look & AFT Look \\
\hline$<2 \mathrm{~km}$ & $0.02 \pm 0.76$ & $0.02 \pm 0.74$ \\
$2-5 \mathrm{~km}$ & $-0.10 \pm 0.85$ & $0.17 \pm 0.84$ \\
$>5 \mathrm{~km}$ & $-0.13 \pm 0.87$ & $0.13 \pm 1.05$ \\
\hline
\end{tabular}

the GrOAWL measurements and the dropsonde observations over the course of the experiment.

Although numerical weather prediction models will likely assimilate line-of-sight velocities rather than wind vectors, it is useful to examine wind vectors computed by combining the two looks from the GrOAWL measurements. Figure 7 shows wind vectors from a portion of the June 21 flight computed from the two looks. Also shown are the dropsonde measurements represented by dots attached to the wind bars. A turn at the end of the racetrack pattern occurred between 18:40 and $18: 50$, as shown by the wind-field symmetry as the lidar re-samples the same region.

An important goal for the AOVT test program was validation of OAWL instrument sensitivity as represented in models used to predict performance of an OAWL-type instrument in space. To examine sensitivity, we attempted to characterize the backscatter environment for each flight from the GEOS-5 model analyses, Aeronet measurements, and lidar observations obtained at the same time of year during previous campaigns. Based on our estimates of backscatter during the flights while accounting for some degradation of the GrOAWL measurements from the harsh platform environment, we determined that our instrument model accurately estimates space-based performance for an OAWL instrument. Further work is currently being carried out in collaboration with NCAR to use HSRL measurements to more precisely characterize the scattering environment during GrOAWL surface-based operation.

\section{DISCUSSION AND FUTURE WORK}

Results from the AOVT campaign provide strong evidence that the OAWL concept is suitable for measurement of winds from space. Advantages of

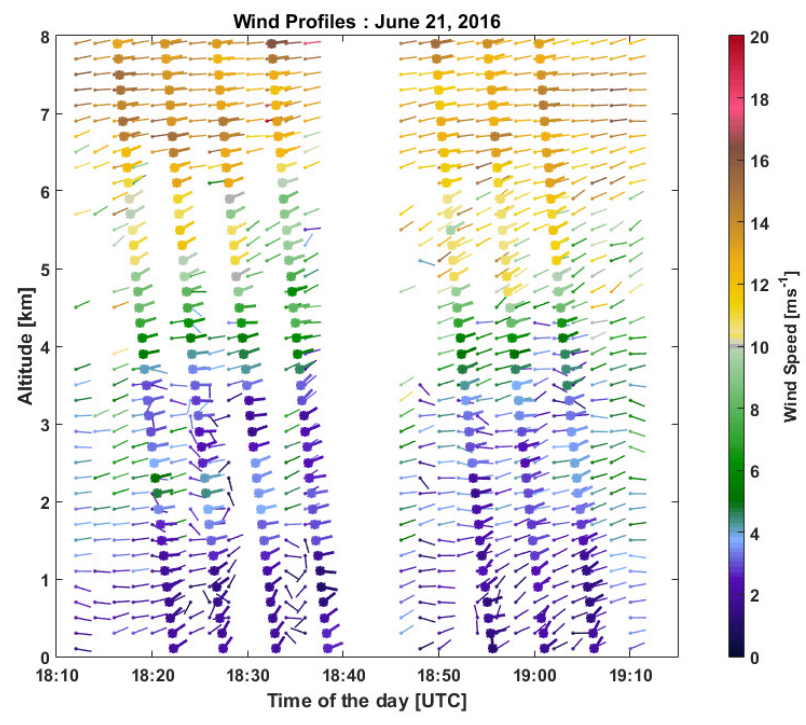

Figure 7: Wind vectors computed from June 21

GrOAWL and dropsonde observations.

the OAWL design include relaxed specifications on laser pulse-to-pulse frequency stability, high transmission, capability to extract very weak signals by averaging, potential for inclusion in a single transmitter/nested receiver design to measure winds from both aerosol and molecular scatterers, and potential for a combined HSRL/Doppler lidar. Projected next steps for GrOAWL include modification for deployment on the NASA DC-8, utilization for Aeolus Calibration and Validation, and application for airborne science flights studying dynamics and transport.

\section{ACKNOWLEDGEMENTS}

We thank the NASA Earth Science Venture Technology Program, the Ball hardware team, and the NASA WB-57 group for their valuable support.

\section{References}

[1] Baker, W. E., et al, 2013: Lidar-Measured Wind Profiles: The Missing Link in the Global Observing System, Bull. Amer. Meteor. Soc., 95, 4, 543-564.

[2] Riishojgaard, L. P., et al, 2012: Observing System Simulation Experiments for a global observing wind sounder, Geophy. Res. Lett. 39, Article L17805.

[3] Tucker, S. C., R. M. Hardesty, S. Baidar, and C. Weimer, 2016: The ATHENA-OAWL Venture Tech Instrument. Proc. $18^{\text {th }}$ Coherent Laser Radar Conference, Boulder, CO, June 26-July 1. 